\title{
Régis REVENIN (coord.), Hommes et masculinités de 1789 à nos jours. Contributions à l'histoire du genre et de la sexualité en France
}

Paris, Éditions Autrement, 2007, 295 pages

\section{Bruno Benvindo}

\section{OpenEdition}

\section{Journals}

\section{Édition électronique}

URL : https://journals.openedition.org/clio/9737

DOI : 10.4000/clio.9737

ISSN : $1777-5299$

Éditeur

Belin

Édition imprimée

Date de publication : 1 mai 2010

ISSN : 1252-7017

\section{Référence électronique}

Bruno Benvindo, "Régis revenIN (coord.), Hommes et masculinités de 1789 à nos jours. Contributions à I'histoire du genre et de la sexualité en France », Clio. Femmes, Genre, Histoire [En ligne], 31 | 2010, mis en ligne le 17 juin 2010, consulté le 21 septembre 2021. URL : http://journals.openedition.org/clio/ 9737 ; DOI : https://doi.org/10.4000/clio.9737

Ce document a été généré automatiquement le 21 septembre 2021.

Tous droits réservés 


\section{Régis REVENIN (coord.), Hommes et masculinités de 1789 à nos jours. Contributions à l'histoire du genre et de la sexualité en France}

Paris, Éditions Autrement, 2007, 295 pages

\section{Bruno Benvindo}

1 Dès 1983, à l'occasion du colloque Une histoire des femmes est-elle possible? qui allait marquer le véritable départ de l'histoire des femmes en France, Alain Corbin soulignait la nécessité de se pencher aussi sur les "mentalités masculines", afin que puisse émerger une « histoire sexuée qui couple ses analyses $»^{1}$. L'auteur des Filles de noce resta longtemps peu entendu. Cette dernière décennie a pourtant vu une nette évolution s'amorcer, les masculinités françaises devenant peu à peu un champ d'enquête historique à part entière. Si bien que près d'un quart de siècle après l'appel du colloque de Saint-Maximin, un volume rassemble pas moins de dix-sept contributions sur ce thème. Ouvrage dont la préface, la boucle étant ainsi bouclée, est signée Alain Corbin.

2 Fruit d'une journée d'études qui s'est tenue en 2006 à la Sorbonne, ce livre coordonné par Régis Revenin présente un large panorama des recherches les plus récentes - les auteur-e-s sont pour la plupart des jeunes chercheur-e-s, rejoints par quelques historien-ne-s plus expérimenté-e-s - sur les masculinités françaises à l'époque contemporaine. Il est introduit par un très utile chapitre historiographique de Judith Surkis, historienne d'Harvard à qui l'on devait déjà un ouvrage important au cœur des thématiques abordées $\mathrm{ici}^{2}$. Sa contribution situe le présent volume dans un champ de recherche international, faisant le point sur l'évolution de l'histoire des masculinités par le biais d'un aller-retour entre historiographies anglo-saxonne et française. Hommes et masculinités de 1789 à nos jours se décline ensuite en cinq volets thématiques, analysant les masculinités au croisement de ces autres rapports de pouvoir et ancrages identitaires que sont la sexualité, le travail, la guerre, la religion et la « race». Se dévoilent ainsi, au fil des différents articles, les processus structurant les modèles 
masculins normatifs comme les fissures qui les lézardent, rappelant à quel point le masculin est une construction sociale instable, plurielle, contingente, quotidiennement réinventée.

3 Arrivé au terme de l'ouvrage, nul ne peut douter que l'étude des masculinités, qui constituaient jusqu'il y a peu un angle mort de la recherche historique en France, y est désormais en plein essor ${ }^{3}$. Le retard historiographique par rapport au monde anglosaxon, mais également vis-à-vis de pays comme l'Italie ou l'Allemagne, est à l'évidence en voie d'être comblé. Ce constat s'accompagne néanmoins d'un regret : celui que ces recherches, indéniablement neuves pour la France, ne soient pas ici - à l'exception notable de certains articles tels ceux de Michael Sibalis ou de Régis Revenin davantage mises en relation avec les acquis historiographiques des autres pays européens, et ainsi intégrées dans une perspective internationale.

Révélant un moment historiographique, ces actes de colloque semblent parfois - mais c'est là évidemment (et sans mauvais jeu de mot) la loi du genre - manquer d'un fil rouge. Le lecteur déplorera peut-être que ce livre rassemblant des contributions fort diverses ait été construit sans véritable perspective intégrée ou élaboration collective plus problématisée où les chapitres se répondraient davantage. De même qu'il regrettera l'absence d'une conclusion générale. Mais cet aspect quelque peu composite reflète avant tout l'état des connaissances: certains thèmes comme l'homosexualité masculine sont désormais bien explorés, tandis que d'autres, telle l'hétérosexualité, restent des terrae incognitae. De même, si l'on se réjouira de découvrir ici de passionnantes pages sur l'articulation entre masculinité et "race ", on s'étonnera que les catégories d'âge ne soient, elles, qu'à peine évoquées. Ce déséquilibre se traduit également dans la chronologie : la Seconde Guerre mondiale est abondamment traitée, alors que rien n'est dit du conflit 1914-1918 ou de la guerre d'Algérie. La période s'étalant de 1945 à nos jours apparait d'ailleurs comme le parent pauvre de ce volume qui n'aborde qu'à peine ces décennies pourtant décisives de l'histoire des masculinités modernes. De nombreux chantiers restent donc ouverts. Ce qui n'est finalement pas la moindre des vertus de ce livre qui - pour reprendre les mots de celui qui l'a dirigé « n'a [pas] la prétention d'être l'Histoire des hommes et des masculinités dans la France contemporaine » (p. 5). L'heure, à l'évidence, n'est pas encore à la synthèse.

Hommes et masculinités de 1789 à nos jours a l'immense mérite, on l'aura compris, d'ouvrir de multiples pistes de réflexion. Cela fait de cet ouvrage foisonnant et novateur une référence incontournable, un point de passage obligé pour ceux et celles qui écriront les masculinités en France, mais aussi pour chacun-e se préoccupant d'histoire des femmes, du genre et de la sexualité. Démonstration est faite, si besoin en était encore, que l'histoire des hommes et celle des femmes ne peuvent se passer l'une de l'autre. Le sous-titre du volume ne ment pas : c'est bien de Contributions à l'histoire du genre et de la sexualité dont il s'agit ici. 


\section{NOTES}

1. Les actes du colloque ont été publiés l'année suivante; Alain Corbin, «Le 'sexe en deuil' et l'histoire des femmes au XIX ${ }^{\mathrm{e}}$ siècle ", dans Michelle Perrot (dir.), Une histoire des femmes estelle possible ?, Paris, Rivages, 1984, respectivement p. 142 et p. 147.

2. Judith Surkis, Sexing the Citizen: Morality and Masculinity in France, 1870-1920, Ithaca, Cornell University Press, 2006. Voir le compte rendu de F. Rochefort plus loin.

3. On verra aussi Christopher Forth \& Bertrand Taithe (eds), French Masculinities: History, Culture and Politics, Basingstoke, Palgrave Macmillan, 2007. 